\title{
Accessing Tacit Knowledge in the Pediatric Pain e-Mail Archives
}

\author{
Qiufen Qi, Qigang Gao and Michael Shepherd \\ Faculty of Computer Science \\ Dalhousie University \\ Halifax, NS, Canada B3H1W5 \\ \{qiufen,qggao,shepherd\}@cs.dal.ca \\ G. Allen Finley \\ Departments of Anaesthesia \& Psychology \\ Dalhousie University, Halifax, NS, Canada \\ allen.finley@dal.ca
}

\begin{abstract}
The Pediatric Pain Mailing List (PPML) is an international Internet-based forum for informal discussion of any topic related to pain in children. There are now over seven hundred members, including clinicians, researchers and patients from at least forty countries on six continents. Currently, the archive contains more than ten thousand messages. The goal of this research is to provide a means by which the tacit knowledge captured in the archive can be organized and accessed in a fruitful manner. This involves the threading together of e-mails such that the threads represent asynchronous discussions and question-answer sessions and then clustering these threads hierarchically such that the PPML archives can be browsed easily.
\end{abstract}

\section{Introduction}

The existence of pediatric pain has been recognized widely in the past fifteen years. Although knowledge about pediatric pain has accumulated over this period, it is still under-treated. Many pediatric pain problems are relatively rare and it is impossible for even a pediatric pain specialist to have personal experience with all possible symptoms and syndromes. In addition, clinical research has been limited and there is little in the medical and scientific literature to support clinical decisionmaking. Therefore, the Pediatric Pain Mailing List (PPML) has become an important valuable information resource for clinicians, researchers and patients.

The Pediatric Pain Mailing List (PPML) is an international Internet-based forum for informal discussion of any topic related to pain in children. It is a list server that permits individuals to post/reply to e-mail messages that are then sent to all the subscribers and the messages themselves are archived at the central server. It was developed ten years ago to promote informal discussions and information exchanges among pediatric professionals [3]. The subscribers to PPML include professionals from different clinical and research disciplines and laymen, such as medical students, pediatric patients and their parents. There are now over seven hundred members from at least forty countries on six continents. Currently, the archive contains more than ten thousand messages.

Over these ten years, a vast amount of tacit knowledge about pediatric pain has been captured in the PPML archives. Tacit knowledge is what the knower knows, knowledge that is derived from experience [11]. This tacit knowledge is captured and shared through e-mail discussions carried on amongst clinicians, researchers and patients through the PPML. As such, the PPML archives have become a repository of knowledge about pediatric pain.

Unfortunately, this knowledge is not readily accessible. The messages are stored in a raw e-mail format and are not organized in any manner. This has made it difficult to provide the subscribers with information retrieval and knowledge discovery from the archive. In order to provide access to this tacit knowledge, a thread-based method for clustering the messages into hierarchical categories was developed. The stored e-mail messages were first cleaned, then organized into discussion threads. These discussion threads were then clustered hierarchically using repeated applications of the $k$-means algorithm [17]. The resulting hierarchy was evaluated against categorizations by two human experts and there were no significant differences found between the results of these two evaluations. 
A Web-based interface allows the subscribers topdown browsing of the hierarchy and thus access to the tacit knowledge in the archives.

Section 2 of this paper discusses the PPML within the context of tacit and explicit knowledge. Section 3 describes the creation of threads of e-mails and their clustering into a hierarchical organization. Section 4 describes the evaluation of the resulting clustering against that of human experts. Section 5 discusses results and section 6 summarizes this paper and discusses future work on linking the PPML to the explicit knowledge found in the published literature.

\section{Tacit-Explicit knowledge framework}

Polanyi [14] introduced the notions of tacit knowledge and explicit knowledge and Nonaka [13] used these notions to formulate a theory of organizational learning. Polanyi's and Nonaka's work can be used to frame the research of the PPML research project, i.e., to frame the knowledge held in the PPML archives and the methods being developed to access this knowledge.

Tacit knowledge is what the knower knows and is derived from experience whereas explicit knowledge is represented by some artifact such as a document or journal article [11]. Within the PPML, the tacit knowledge of clinicians, researchers and patients with respect to pediatric pain is captured and transformed into explicit knowledge as represented by threads of e-mail messages that represent conversations and discussions on particular topics. This transformation of knowledge is represented by Figure 1, as adapted from Nonaka. The four processes by which knowledge is transformed are socialization, externalization, combination and internalization. The spiral indicates the continuous nature of this transformation among the four processes. The processes are:
- Socialization is the transformation of tacit knowledge to tacit knowledge as experienced in face-to-face meetings and the communication is synchronous in nature;

- Externalization is the transformation of tacit knowledge to explicit knowledge as might take place in responding to questions. In this instance, the communication may be asynchronous in nature as found in list server discussion groups;

- Combination is the transformation of explicit knowledge to explicit knowledge. Once knowledge as been articulated and captured in a persistent form, it can be organized into a hierarchically organized set of categories where the organization itself reflects knowledge of the domain;

- Internalization is the transformation of explicit knowledge to tacit knowledge. Access to an organized or structured set of documents that is a representation of explicit knowledge helps the user to more quickly internalize the knowledge, thus converting the explicit knowledge into their own tacit knowledge.

Within the PPML, there is no accommodation for the process of socialization, i.e., the transformation of tacit knowledge to tacit knowledge as there is no means for synchronous communication as might be found in chat rooms or supported by groupware. The PPML does, however, support the process of externalization as tacit knowledge is elicited as people ask questions through the list server and those with tacit knowledge respond, asynchronously. The tacit knowledge captured is that of the responder, i.e., what the responder knows from experience. The post-reply dialog transforms this tacit knowledge into explicit knowledge. These e-mail discussions or threads are artifacts of explicit knowledge.

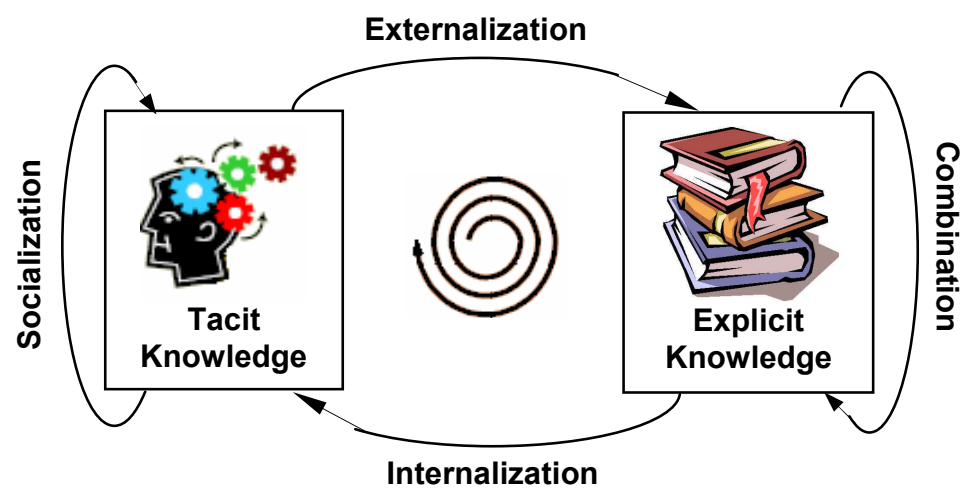

Figure 1. Knowledge transformation processes 
Unfortunately, the knowledge accumulated in the PPML archives was very difficult to access as it was not organized in any manner other than chronologically. Therefore, this research was to transform this explicit knowledge by tying together e-mail posts and replies (conversations) into e-mail threads and organizing these threads into an hierarchically organized set of clusters of treads (combination transformation). This hierarchical organization is the "valued-added" by this explicit to explicit knowledge transformation. This hierarchically organized structure then permits users to browse through the knowledge captured in the PPML archives and to internalize this explicit knowledge (internalization).

\section{Methodology}

In order to make the knowledge in the PPML archives accessible, it was necessary to bring e-mails together to form threads, i.e., identify originating postings and the chain(s) of e-mail responses to those postings, and then to form a hierarchical clustering of those threads.

\subsection{Cleaning and threading}

In order to do this, the first step was to clean the data. This involved removing duplicate messages as identified by subject and date/time stamps, removing responses that were generated automatically by "vacation" mail programs, removing other "junk" e-mails, and by removing unnecessary content of the messages themselves. This unnecessary content included nontextual material such as images that would not be used in the clustering process and included original messages that were more than ten lines long as these would skew the clustering process. The initial stage of this cleaning was done manually until patterns were recognized and then programs were written to clean the data based on these patterns.

After data cleaning, the messages were threaded based on time stamps and subject headings. Those messages that had a blank subject field were processed based on the included original messages to which they had replied. Lewis and Knowles [10] found that threading based on subject field content and on included original messages gave reasonably high thread coherence.

Prior to cleaning and threading, there were 6939 messages in the archive, from 1993 to 1999. After cleaning and threading, this was reduced to 4033 messages in 1289 threads. A sample thread is shown in the Appendix, with all identifying information removed.

\subsection{Clustering of threads}

Text clustering has been investigated in many different areas of text mining and information retrieval. It is an unsupervised learning process of grouping documents into clusters so that the documents within a cluster have high similarity with one another, but are very dissimilar to the documents in the other clusters [4]. It was initially used as a way of improving the efficiency of best match searching in information retrieval systems [20]. More recently, it has been used in browsing a large collection of documents [2] and in organizing the results returned by a search engine to help users find relevant documents more quickly [23]. Document clustering has also been used to automatically generate hierarchical clusters of documents [7].

Note that text clustering is a different task than text categorization or classification. Text categorization assigns pre-defined categories to new documents, based on the likelihood suggested by the labeled documents in the training set $[16,21]$. Text categorization is a supervised task involving pre-defined category labels, while text clustering is an unsupervised task and there is no pre-defined category label available. Most other projects involving e-mail messages, such as that of Itskevitich [5], use text classification of the messages (not the threads) in order to filter incoming e-mail messages into pre-defined folders.

In this project, clustering (as opposed to classification) was applied to the e-mail threads in order to organize these artifacts of explicit knowledge into a hierarchical structure. The hierarchical structure discovered in this manner should reflect the hierarchical structure of the knowledge captured in the list server archives and permit users to browse and query the archive in a fruitful manner.

3.2.1. $k$-means clustering. Each thread is treated as though it was a contiguous document and is represented by a weighted term vector. The original messages that are embedded in the reply messages are removed. The terms from the remaining text are checked against an augmented list of stop words [19]. If not on the stop list, they are matched against a synonym dictionary manually created by a pediatric pain specialist. These terms are then stemmed using Porter's algorithm [15]. The stemmed terms are assigned tf.idf (term frequency - inverse document frequency) weights [8] so that the thread vectors consist of weighted stemmed terms. 
These thread vectors are clustered hierarchically using repeated applications of the $k$-means algorithm. Over the years, many clustering techniques have been developed. Two recent papers $[1,6]$ offer a comprehensive survey of different clustering algorithms and applications. The $k$ means text clustering algorithm [17] is a top-down or divisive algorithm that partitions the dataset into a nonhierarchical set of clusters. The basic $k$-means algorithm is:

1. Randomly select $k$ data objects from the whole dataset;

2. Treat these data objects as the initial cluster centroids;

3. Assign each of the remaining data objects to the most similar cluster, based on the similarity of the object with the cluster centroid;

4. Update the centroid of the cluster;

5. Repeat steps 3 and 4 until the centroids are stable.

Repeated application of the $k$-means algorithm produces a hierarchy of arbitrary breadth and depth. Assuming that the dataset of threads is the initial cluster, the algorithm as applied in this project is:

1. Chose a cluster at the current level (starting with the initial cluster);

2. Apply $k$-means algorithm to this cluster, generating $k$ sub-clusters;

3. Repeat until all the clusters at the bottom level of the hierarchy contain $k$ or few threads.
By setting $k$ equal to $2,4,6,8$ or 10 , we are able to produce hierarchies of different breadths and depths. In general, the larger the value of $k$, the broader and shallower is the resulting hierarchy as the algorithm will subdivide each cluster into $k$ sub-clusters at each level. For $k=2$, the resulting hierarchy is narrow and quite deep, and a perfectly balanced hierarchy would have a depth of approximately 10 ( $\log _{2}$ of 1289 threads). For $k=10$, the resulting hierarchy is quite broad and shallow, and the perfectly balanced hierarchy would have a depth of approximately 3 ( $\log _{10}$ of 1289 threads). Those hierarchies that are not perfectly balanced (most cases) will have some sub-trees that are quite shallow and some sub-trees that are much deeper, but will still be $k$ sub-trees wide. In this project, hierarchies were generated and stored for all five of these values of $k$ and the user is given the choice of which hierarchy they chose to browse.

Figures 2 and 3 are example snapshots of the resulting system, showing the root (Pediatric pain) and the first level of sub-clusters. Note that in Figure 2, the user has chosen to view the hierarchy generated with $k=6$, and in Figure 3 with $k=8$. Thus, the hierarchy as shown in Figure 2 has 6 sub-clusters at each level and the hierarchy shown in Figure 3 has 8 sub-clusters at each level. Each cluster is represented by the fifteen highest ranked terms in the vectors in that cluster. A single thread may be viewed by traversing to the bottom of the hierarchy and clicking on a thread.

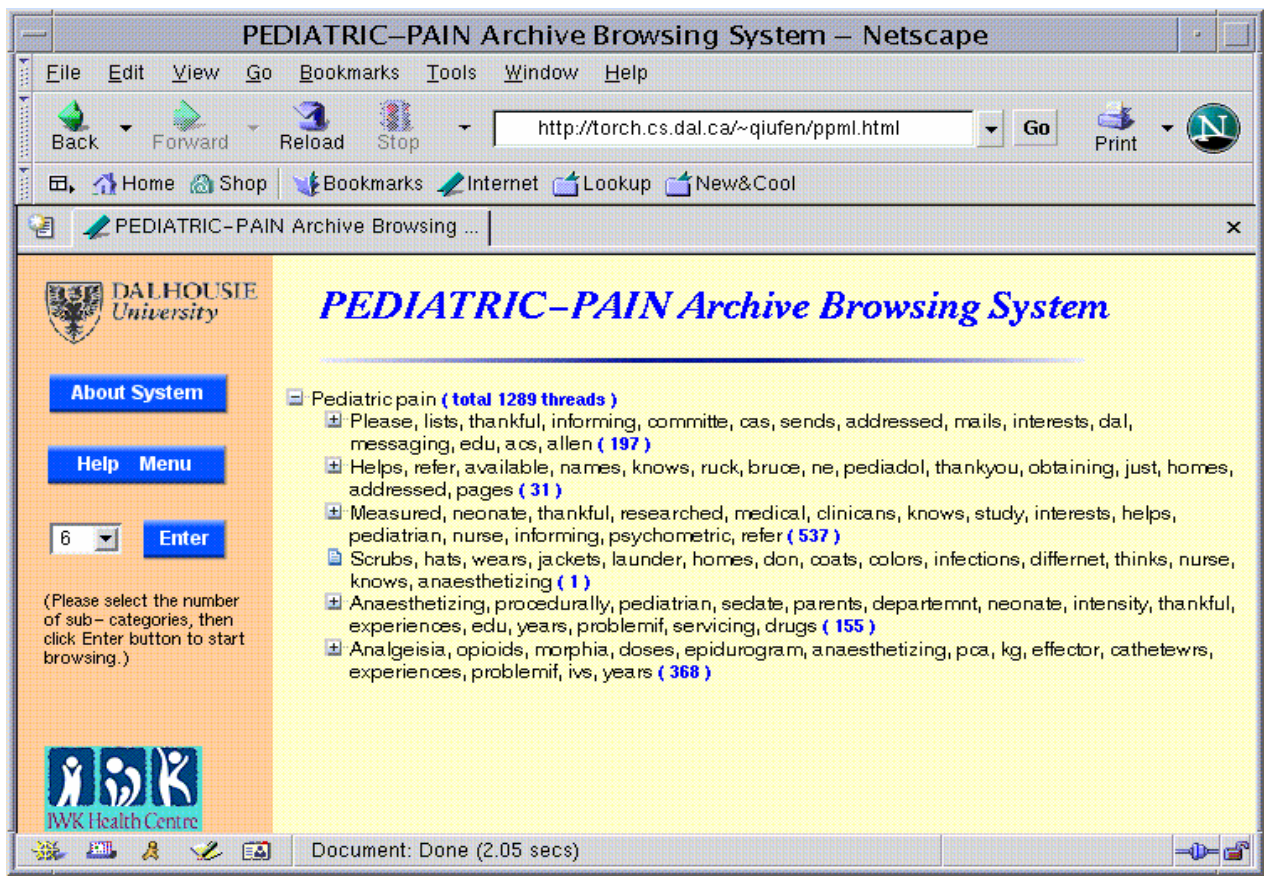

Figure 2. Root and top level of clusters for $k=6$ 


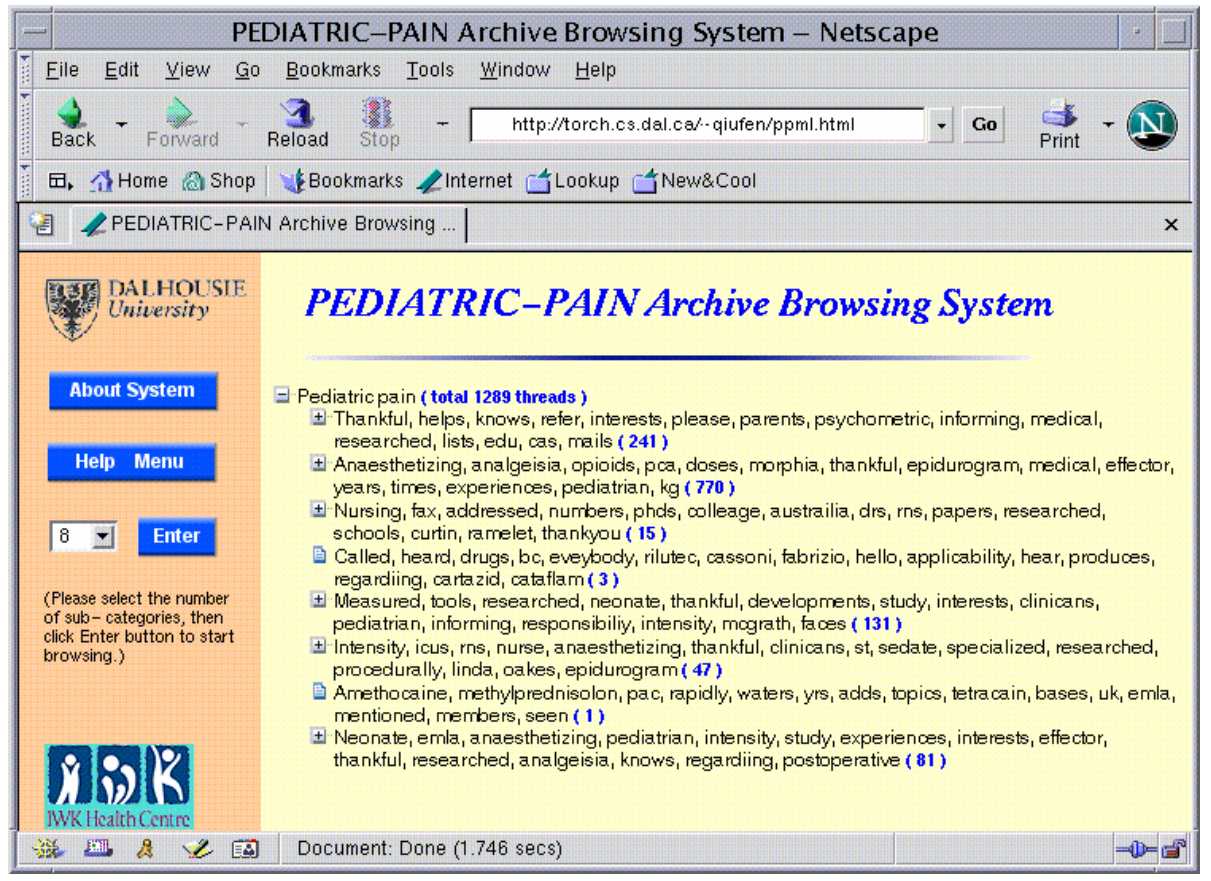

Figure 3. Root and top level of clusters for $k=8$

\section{Evaluation methodology}

A number of experiments were run to investigate both effectiveness and efficiency of the algorithm. Efficiency of the algorithm addresses time and space considerations. Effectiveness of the algorithm addresses the quality of the resulting hierarchical clustering. As access to tacit knowledge is the focus of the research as presented in this paper, only the quality of the resulting hierarchy of clusters of threads is discussed in this paper.

Although a full user study has not yet been done, the resulting hierarchies were compared against clustering performed by two experts in the field of pediatric pain. One hundred threads were selected randomly from the dataset. These threads were presented to the two experts and they were asked to independently partition these threads into a flat (one-level) set of clusters. The results are shown in Tables 1 and 2. As can be seen, the resulting sets of clusters are quite different. Expert 1 generated 13 clusters and Expert 2 generated 17 clusters and the class labels the experts assigned are quite different. The extent of this difference between the two sets of clusters created by the experts was measured using the information theoretic measure of redundancy [22] as it was applied in earlier experiments by Tague and Shepherd [18].

The Redundancy $(\mathrm{X}, \mathrm{Y})$ is the proportion of uncertainty about $\mathrm{X}$ that is removed by knowing $\mathrm{Y}$, and is given by:

$$
\text { Redundancy }(X, Y)=(H(X)-H(X \mid Y)) / H(X)
$$

where:

$\mathrm{H}(\mathrm{X})=$ the entropy or uncertainty of $\mathrm{X}$

$\mathrm{H}(\mathrm{X} \mid \mathrm{Y})=$ the uncertainty of $\mathrm{X}$ given that $\mathrm{Y}$ is known

In this instance, $\mathrm{X}$ and $\mathrm{Y}$ represent the two sets of clusters generated by the experts. The measure is asymmetrical and the calculated redundancy measures are:

$$
\begin{aligned}
& \mathrm{R}(\text { Expert-1, Expert-2) }=0.51 \\
& \mathrm{R}(\text { Expert-2, Expert-1) }=0.44
\end{aligned}
$$

Therefore, the redundancy between the two expertgenerated sets of clusters is between 44 and 51 percent and the sets of clusters have some similarity. 
Table 1. Clusters and labels created by expert 1

\begin{tabular}{clc}
$\begin{array}{c}\text { Cluster } \\
\text { ID }\end{array}$ & \multicolumn{1}{c}{ Label } & $\begin{array}{c}\text { Number of } \\
\text { Threads }\end{array}$ \\
1 & adverse effects of a treatment or medication, monitoring requirements & 6 \\
2 & advice on medications or treatment technique for a particular CONDITION & 33 \\
3 & announcement of a publication or event & 9 \\
4 & assessment methods for a particular condition & 2 \\
5 & availability and benefits of a nonstandard drug compound & 3 \\
6 & availability and validation of a particular assessment tool & 11 \\
7 & contact information or other information about a specific person & 3 \\
8 & dosage or adverse effects or technique for a medication or other treatment & 9 \\
9 & information on a condition: description, etiology, prognosis & 4 \\
10 & job description, posting of job or fellowship & 3 \\
11 & miscellaneous & 3 \\
12 & other newsgroups and listservs & 2 \\
13 & policies, guidelines, protocols, algorithms, quality assurance, supervision, competency & 12
\end{tabular}

Table 2. Clusters and labels created by expert 2

$\begin{array}{clc}\begin{array}{c}\text { Cluster } \\ \text { ID }\end{array} & \text { Label } & \begin{array}{c}\text { Number of } \\ \text { Threads }\end{array} \\ 1 & \text { Assessment } & 10 \\ 2 & \text { Musculoskeletal } & 2 \\ 3 & \text { sedation \& procedures } & 4 \\ 4 & \text { oral drugs } & 5 \\ 5 & \text { miscellaneous /irrelevant/out-of-date } & 21 \\ 6 & \text { neuropathic pain } & 9 \\ 7 & \text { regional analgesia } & 10 \\ 8 & \text { postoperative pain } & 5 \\ 9 & \text { intravenous opioids } & 11 \\ 10 & \text { psychology } & 5 \\ 11 & \text { visceral pain, bowel function, etc } & 2 \\ 12 & \text { topical analgesia, EMLA } & 2 \\ 13 & \text { everyday pain } & 1 \\ 14 & \text { NMDA antagonists, ketamine } & 1 \\ 15 & \text { resources } & 8 \\ 16 & \text { administration } & 3 \\ 17 & \text { burns } & 1\end{array}$

Unfortunately, this measure could not be used to measure the redundancy between the manually-created sets of clusters and the computer-generated hierarchy because of the difference in the structures (hierarchical vs. non-hierarchical). Therefore, a different measure had to be used to evaluate the hierarchical clustering.

Several measures have been used in the literature to measure the quality of clusters. They include "entropy", "overall similarity", " $F$-measure" and more. Among them, the $F$-measure is more suitable for measuring the effectiveness of hierarchical clustering [17]. The $F$ - measure is a traditional effectiveness measure in information retrieval area, and was recently introduced to document clustering [9].

For each humanly generated set of clusters, an $F$ measure value was calculated for each cluster and an overall $F$-measure was then calculated as a measure of the hierarchical clustering as compared to that expert's clustering. For this experiment, the $k$-means clustering was done on the same 100 threads that the human experts clustered. The evaluation method was as follows: 
1. Treat each manually created cluster, $T$, as though a query had been asked and the threads in that cluster represent the set of threads relevant to that query;

2. The $k$-means cluster $C$ that corresponds to $T$ will be formed somewhere in the cluster hierarchy and will include all clusters in the sub-tree from that point;

3. To find $C$, traverse the hierarchy and at each node in the hierarchy calculate the $F$-measure for $C$;

4. The cluster $C$ with the highest $F$-measure is the cluster corresponding to humanly-generated cluster $T$.

For any manually generated cluster $T$ and cluster $C$ :

$$
\begin{aligned}
& \text { Precision }(C, T)=N /|C| \\
& \text { Recall }(C, T)=N /|T| \\
& F \text {-measure }=2 P R /(P+R)
\end{aligned}
$$

where $N$ is the number of threads common to both $T$ and $C$

$|C|$ is the number of threads in cluster $C$,

$|T|$ is the number of threads in the manually generated cluster $T$

$P$ stands for precision and $R$ for recall.

The overall $F$-measure is used to reflect the quality of the whole hierarchy. The overall $F$-measure is the average weighted $F$-measure for all the clusters in a humanly generated clustering and is defined to be: where $S$ is the set of humanly generated clusters,

$|T|$ is the number of threads in cluster $T$

$F(T)$ is the $F$-measure for category $T$.

This overall $F$-measure reflects the quality of the resulting hierarchy, i.e., the closeness between the resulting hierarchy and manually generated clusters. It ranges from 0 to 1 where 1 is the best quality.

\section{Results}

The evaluation was conducted as described above for all five values of $k$ for the $k$-means generated hierarchical clusters. For any one value of $k$, different clusters may be generated, depending on which threads are randomly selected to represent the initial centroids of the clusters being generated. Therefore, for each value of $k$, the algorithm was run ten times with different threads randomly selected to act as initial centroids, resulting in ten different hierarchical classifications.

Figure 4 compares the resulting overall $F$-measure values for the two experts for $k=6$ for each of the ten runs. The bar on the left of each pair represents the $F$-measure for expert 1 and the bar on the right of each pair the result for expert 2. The results of the paired-samples tests $(\mathrm{p}>0.05)$ show that there was no significant difference between the two sets of manually generated clusters on the overall $F$-measures. The average overall $F$-value for expert 1 is 0.47 and for expert 2 it is 0.48 .

$$
\text { Overall } F \text {-measure }=\sum_{T \in S}(|T| * F(T)) / \sum_{T \in S}|T|
$$

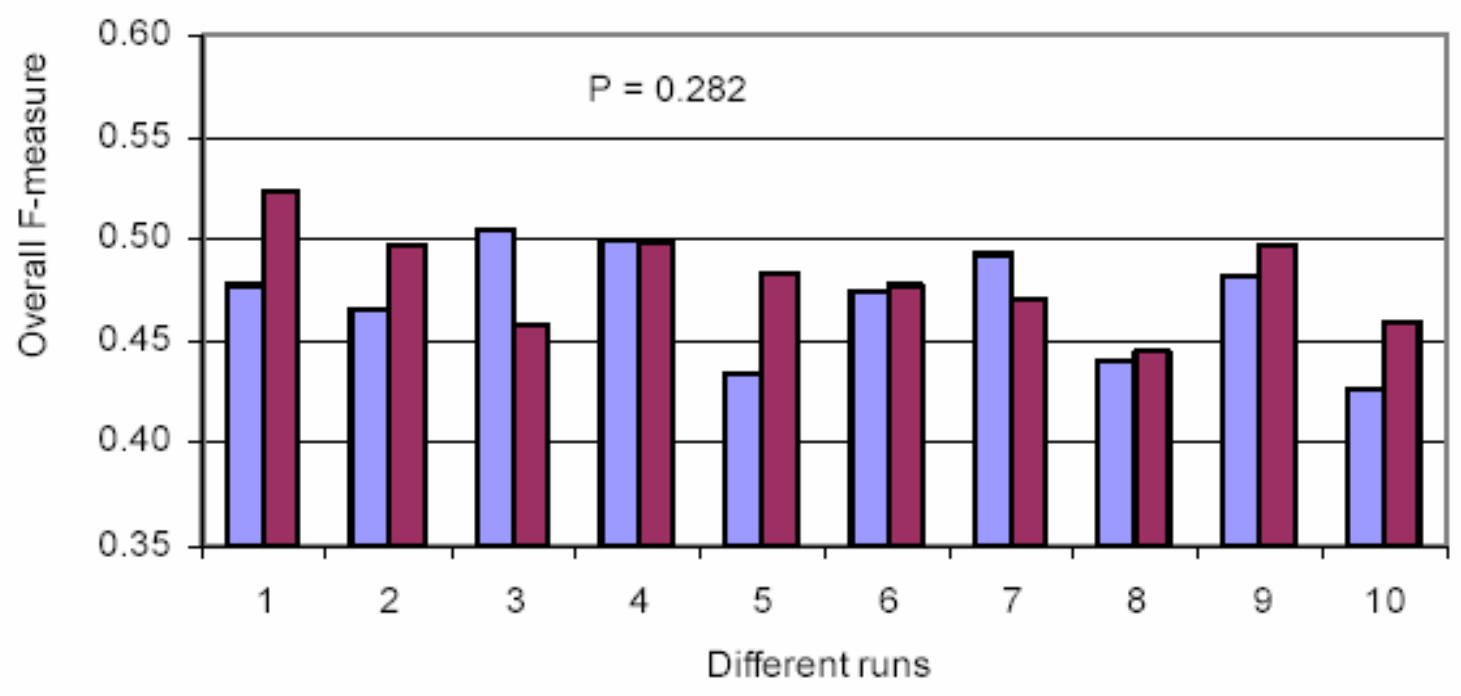

Figure 4. Comparing effects of clusters generated by experts Bar on left of each pair is for expert 1, bar on right for expert 2. 
The redundancy measure has shown that the two manually generated sets of clusters have some amount of similarity between them and when each of these sets of clusters was used to evaluate the quality of the hierarchical clustering, there was no significance difference between the $F$-measure results. From this, one could infer that computer-generated clustering is not that different from either of the two manually generated sets of clusters, and is a reasonably good representation of the organization of the domain of explicit knowledge represented in the threaded dataset.

\section{Summary and future work}

The research reported in this paper is the first stage of a much larger project. To date, we have demonstrated that the tacit knowledge of experts can be captured and transformed into explicit knowledge through asynchronous discussions and question-answering as provided by the PPML. This explicit knowledge is captured as e-mail threads. This explicit knowledge, represented as e-mail threads, can be further transformed through being organized hierarchically to represent the domain knowledge captured in the archives and expressed in the threads and that this hierarchical organization is a reasonably good representation. This explicit domain knowledge can then be browsed or queried by clinician, researchers and patients.

The intent of the overall research project is to link the tacit knowledge captured in the PPML archives to the explicit knowledge as represented by best-evidence publications found in refereed journal and conference papers in service providers such as PUBMED [12]. As people browse the PPML, they may wish to discover bestevidence publications to either support or refute knowledge expressed in the PPML. The project will extract the main concepts from the thread or cluster of threads and use these concepts to search PUBMED automatically, returning any documents found.

Similarly, publications are often somewhat "dated". Therefore, people reading documents from PUBMED should be able to link directly to the PPML to discover if there are any recent e-mail discussions concerning a particular topic. The main concepts from the PUBMED article will be used to search for relevant threads in the PPML.

Full user evaluations will be done to determine the efficacy of this method of discovering and linking tacit knowledge and explicit, best-evidence knowledge.

\section{Acknowledgements}

We would like to thank Dr. Carl von Baeyer, Department of Psychology, University of Saskatchewan, for manually creating one of the sets of clusters for evaluation purposes. The second set was created by Dr. Allen Finley, Departments of Anaesthesia and Psychology, Dalhousie University, one of the authors of this paper.

\section{References}

[1] Berkhin P., Survey of Clustering Data Mining Techniques, Technical Report, Accrue Software, Inc, 2002.

[2] Cutting, D.R., Karger, D.R., Pederson, J.O. and J.W. Tukey, "Scatter/Gather: A Cluster-based Approach to Browsing Large Document Collections", Proc. of the $15^{\text {th }}$ Annual International ACM SIGIR Conf. on Research and Development in Information Retrieval, Copenhagen, Denmark, 1992, pp. 318-329.

[3] Finley, G.A. and P.J. McGrath, "The PEDIATRIC-PAIN Mailing List - An Electronic Interdisciplinary Team", $3^{\text {rd }}$ International Symposium on Pediatric Pain, 1994.

[4] Han, J. and M. Kamber, Data Mining: Concepts and Techniques. Morgan Kaufmann Publishers, New York, 2001.

[5] Itskevitch, J., Automatic Hierarachical E-Mail Classification Using Association Rules, Master of Science Thesis. Simon Fraser University, 2001.

[6] Jain, A.K., Murty, M.N. and p.J. Flynn, Data Clustering: A Review, ACM Computing Surveys, 1999, 31(3), pp. 264-323.

[7] Koller, D. and M. Sahami, "Hierarchically Classifying Documents Using Very Few Words", Proc. of the $14^{\text {th }}$ International Conf. on Machine Learning, Nashville, Tennessee, 1997, pp. 170-178.

[8] Korfhage, R.R., Information Storage and Retrieval, Wiley Computer Publishing, New York, 1997.

[9] Larsen, B. and C. Aone, "Fast and Effective Text Mining Using Linear-time Document Clustering", Proc. of the $5^{\text {th }} A C M$ SIGKDD International Conf. on Knowledge Discovery and Data Mining, San Diego, California, 1999, pp. 16-22.

[10] Lewis, D.D. and K.A. Knowles, "Threading Electronic Mail: A Preliminary Study", Information Processing and Management, 1997, 33(2), pp. 209-217.

[11] Marwick, A.D. "Knowledge Management Technology", IBM Systems Journal, 2001, 40(4).

[12] National Library of Medicine.

[http://www.pubmedcentral.nih.gov/] Available June 9, 2004. 
[13] Nonaka, I. and H. Takeuchi, The Knowledge Creating Company. Oxford University Press, Oxford, 1995.

[14] Polanyi, M., “The Tacit Dimension”, In Prusak, L. (Ed.), Knowledge in Organizations, Butterworth-Heinemann, Woburn, MA, 1997, 135-146.

[15] Porter, M., [http://www.tartarus.org/ martin/PorterStemmer/] Available June 9, 2004

[16] Sebastiani, F., "Machine Learning in Automated Text Categorization", ACM Computing Surveys 2002 34(1), pp. 1-47.

[17] Steinbach, M., Karypis, G. and V. Kumar, "A Comparison of Document Clustering Techniques", Proceeding of Text Mining Workshop, KDD, 2000.

[18] Tague, J. and M. Shepherd, "Redundancy as a measure of Classification Similarity", Journal of Informatics, 2(3), 1978, pp. 123-134.
[19] WAIS (Version: iubio-wais-8-b5)

[http://www.iiasa.ac.at/docs/R_Library/libsrchs.html] Available June 9, 2004.

[20] Willett, P., "Recent Trends in Hierarchic Document Clustering: A Critical Review", Information Processing \& Management, 1988, 24(5), pp. 577-597.

[21] Yang, Y., and X. Liu, "A Re-examination of Text Categorization Methods", Proc. of the $22^{\text {nd }}$ annual international ACM SIGIR Conf. on Research and Development in Information Retrieval, New York, 1999, pp. 42-49.

[22] Young, J.F., Information Theory, New York, Wiley, 1971.

[23] Zamir, O., Etzioni, O., Madani, O., and R.M. Karp, "Fast and Intuitive Clustering of Web Documents", KDD, 1997, pp. 287-290.

\section{Appendix - Sample Thread}

\section{Cluster Keywords: Opioids, days, ivs, guillain, meningococcemia, ptosis, spontaneous, agonist, annequin, nubain, relieve, neurologist, nsaids, acetaminophens, reduce ...}

Total 1 thread(s) in this cluster: 1

\section{Thread 1: opioids and meningitis}

Date: Wed, 04 Jan $199516: 54: 48$-0500 (EST)

From: poster

Subject: opioids and meningitis

$\mathrm{X}$ is a 13 month (9.8kg) old boy suffering from acute meningitis

(pneumocoque) treated with IV cefotaxime; at day three, I have been called as pediatric pain consultant to assess X; I have discovered an extreme painfull state: one could not handle or touch him without producing screaming. The child was unable to move spontaneously he looked paralysed by pain and hypertonia ; he also presented a neurological complication : ptosis at the right side.The pain treatment was IV acetaminophen. The first day I have prescribed IV Nalbuphine (weak opioid u antagonist and agonist) 11mg/24h after a loading dose of $1.4 \mathrm{mg}$; Pain at rest has been succesfully relieved but not the mobilisation pain; the dose has been increased at $14 \mathrm{mg} /$ day wihout relieving the pain associated with moving; he has moved spontaneously limbs 2 days later; nalbuphine has been stopped 4 days later. Neurological examination and CT scan have been still normal (except ptosis) during this period. No opioid's side effects have been observed.

What do you think of this case ?

Have you any experience with opioids and acute meningitis ?

Dr Poster, Pediatric pain unit, Poster Hospital 
Date: Wed, 04 Jan $199517: 27: 25$-0500 (EST)

From: first reply

Subject: re: opioids and meningitis

Is there any periosteal involvement? If so an NSAID (ibuprofen or naproxen) may be much more effective than even opioid.

Date: Wed, 04 Jan $199519: 06: 32-0400$

From: second reply

Subject: Re: opioids and meningitis

Poster writes:

$>\mathrm{X}$ is a 13 month (9.8kg) old boy suffering from acute meningitis... sextreme painfull state: one could not handle or touch him without $>$ producing screaming....

$>$ The first day I have prescribed IV Nalbuphine ...

s succesfully relieved but not the mobilisation pain;...

>has moved spontaneously limbs 2 days later; nalbuphine has been stopped 4

>days later. Neurological examination and CT scan have been still normal...

I have used IV morphine for similar severe meningitis pain, with success. I

wouldn't hesitate to use a pure opioid agonist (in conjunction with acetaminophen, NSAID, and/or tricyclics). However, it sounds like you have the situation under control.

Second Reply, Associate Professor, Dept and University

Date: Thu, 05 Jan $199518: 58: 32$-0800 (PST)

From: Third Reply

Subject: Re: opioids and meningitis

I wonder if the problem is not due to severe arachnoiditis that is secondary to the inflammation. I would suggest a trial of steroids in this patient, perhaps in combination with a benzodiazepine to reduce the spasm. Narcotics may reduce the pain but I would not like to keep $x$ on them for too long. Good luck

Third Reply 